\title{
Superscaling analyses of inclusive electron scattering and their extension to charge- changing neutrino cross sections in nuclei
}

Cite as: AIP Conference Proceedings 899, 3 (2007); https://doi.org/10.1063/1.2733028

Published Online: 27 April 2007

A. N. Antonov, M. V. Ivanov, M. K. Gaidarov, E. Moya de Guerra, J. A. Caballero, M. B. Barbaro, J. M. Udias, and P. Sarriguren

\section{ARTICLES YOU MAY BE INTERESTED IN}

Charged-current inclusive neutrino cross sections in the SuperScaling model AIP Conference Proceedings 1722, 030005 (2016); https://doi.org/10.1063/1.4944128

Charged-current quasielastic (anti)neutrino cross sections on ${ }^{12} \mathrm{C}$ with realistic spectral functions including meson-exchange contributions

AIP Conference Proceedings 2075, 070004 (2019); https://doi.org/10.1063/1.5091195

Inclusive Electron Scattering from Nuclei in the Quasielastic Region

AIP Conference Proceedings 967, 83 (2007); https://doi.org/10.1063/1.2834516

Challenge us.

What are your needs for periodic signal detection?

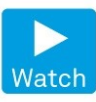

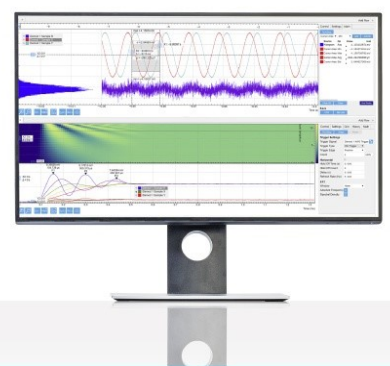

with realistic spectral 


\title{
Superscaling analyses of inclusive electron scattering and their extension to charge-changing neutrino cross sections in nuclei
}

\author{
A.N. Antonov*, M.V. Ivanov*, M.K. Gaidarov*, E. Moya de Guerra ${ }^{\dagger, * *}$, \\ J.A. Caballero ${ }^{\ddagger}$, M.B. Barbaro ${ }^{\S}$, J.M. Udias ${ }^{* *}$ and P. Sarriguren ${ }^{\dagger}$ \\ *Institute for Nuclear Research and Nuclear Energy, Bulgarian Academy of Sciences, Sofia 1784, Bulgaria \\ †nstituto de Estructura de la Materia, CSIC, Serrano 123, 28006 Madrid, Spain \\ ** Departamento de Fisica Atomica, Molecular y Nuclear, Facultad de Ciencias Fisicas, Universidad \\ Complutense de Madrid, Madrid E-28040, Spain \\ † Departamento de Fisica Atomica, Molecular y Nuclear, Universidad de Sevilla, Apdo. 1065, 41080 Sevilla, \\ Spain \\ $\S$ Dipartimento di Fisica Teorica, Università di Torino and INFN, Sezione di Torino, Via P. Giuria 1, 10125 \\ Torino, Italy
}

\begin{abstract}
Superscaling analyses of inclusive electron scattering from nuclei are extended from the quasielastic processes to the delta excitation region. The calculations of $\left(e, e^{\prime}\right)$ cross sections for the target nucleus ${ }^{12} \mathrm{C}$ at various incident electron energies are performed using scaling functions $f\left(\psi^{\prime}\right)$ obtained in approaches going beyond the mean-field approximation, such as the coherent density fluctuation model (CDFM) and the one based on the light-front dynamics (LFD) method. The superscaling properties of the electron scattering are used to predict charge-changing neutrino-nucleus cross sections at energies from 1 to $2 \mathrm{GeV}$. The analyses make it possible to gain information about the nucleon correlation effects on both local density and nucleon momentum distributions.
\end{abstract}

Keywords: inclusive lepton scattering, density distributions, nucleon momentum distributions, nuclear models

PACS: $25.30 .-\mathrm{c}, 21.60 .-\mathrm{n}, 25.30 . \mathrm{Pt}, 21.10 . \mathrm{Ft}$

\section{INTRODUCTION}

An important task of the nuclear theory is to develop methods to study quantities which cannot be explained within the mean-field approximation (MFA) due to the effects of the nucleon correlations. One of quantities which are sensitive to these effects is the nucleon momentum distribution (NMD) in nuclei. In recent years information about NMD has been obtained from the studies of the scaling phenomenon. The concepts of scaling $[1,2]$ and superscaling $[3,4,5,6]$ have been explored in $[4,7]$ for extensive analyses of the $\left(e, e^{\prime}\right)$ world data. Scaling of the first kind (no dependence on the momentum transfer) is reasonably good as expected, at excitation energies below the quasielastic $(\mathrm{QE})$ peak, whereas scaling of second kind (no dependence on the mass number) is excellent in the same region. When both types of scaling behavior occur one says that superscaling takes place. The superscaling analyses of inclusive electron scattering from nuclei for relatively high energies (several hundred $\mathrm{MeV}$ to a few $\mathrm{GeV}$ ) have recently been extended to include not only quasielastic processes, but also the region where $\Delta$-excitation dominates [8].

The superscaling analyses and the present knowledge of inclusive electron scattering allowed one to start stud- ies of neutrino scattering off nuclei on the same basis $([8,9,10,11,12])$.

In this article we follow our method presented in [5] to calculate the scaling function in finite nuclei firstly within the coherent density fluctuation model (e.g., [13]) and also in the light-front dynamics method $[14,15]$. The two methods are used to describe both $y$ - and $\psi^{\prime}$ scaling data (also in the $\Delta$-region) and their applications to analyses of electron and neutrino scattering off nuclei.

\section{SCALING FUNCTION IN THE QUASIELASTIC AND DELTA REGION}

The superscaling behavior of the scaling function was firstly considered within the framework of the relativistic Fermi gas (RFG) model $[3,6,7,16]$ where a properly defined function of the $\psi^{\prime}$-variable was introduced. It was observed that the experimental data have a superscaling behavior in the low- $\omega$ side ( $\omega$ being the transfer energy) of the quasielastic peak for large negative values of $\psi^{\prime}$ (up to $\psi^{\prime} \approx-2$ ), while the predictions of the RFG model are $f\left(\psi^{\prime}\right)=0$ for $\psi^{\prime} \leq-1$. This imposes the consideration of the superscaling in realistic finite systems. 
One of the approaches to do this was developed [5] in the CDFM [13]. It was shown in [5] that the superscaling in nuclei can be explained quantitatively on the basis of the similar behavior of the high-momentum components of the nucleon momentum distribution in light, medium and heavy nuclei which is due to the effects of the short-range and tensor NN correlations in nuclei (see, e.g. [13]).

In the CDFM, the total QE scaling function is expressed by the sum of the proton $f_{\bar{P}}^{Q E}\left(\psi^{\prime}\right)$ and neutron $f_{n}^{O E}\left(\psi^{\prime}\right)$ scaling functions which are determined by the proton and neutron densities $\rho_{p}(r)$ and $\rho_{n}(r)$, respectively [5]:

$$
f_{p(n)}^{Q E}\left(\psi^{\prime}\right)=\int_{0}^{\alpha_{p(n)} /\left(k_{F}^{p(n)}\left|\psi^{\prime}\right|\right)} d R\left|F_{p(n)}(R)\right|^{2} f_{R F G}^{p(n)}\left(R, \psi^{\prime}\right) .
$$

In Eq. (1) the proton and neutron weight functions are obtained from the corresponding proton and neutron densities (normalized to $Z$ or $N$ )

$$
\begin{gathered}
\left|F_{p(n)}(R)\right|^{2}=-\left.\frac{4 \pi R^{3}}{3 Z(N)} \frac{d \rho_{p(n)}(r)}{d r}\right|_{r=R}, \\
k_{F}^{p(n)}=\alpha_{p(n)} \int_{0}^{\infty} d R \frac{1}{R}\left|F_{p(n)}(R)\right|^{2},
\end{gathered}
$$

and $\alpha_{p(n)}=[9 \pi Z(N) / 4]^{1 / 3}$. The RFG proton and neutron scaling functions $f_{R F G}^{p(n)}\left(R, \psi^{\prime}\right)$ have the form given in [5]. Then the total normalized to unity scaling function can be expressed by

$$
f^{Q E}\left(\psi^{\prime}\right)=\frac{1}{A}\left[Z f_{\bar{p}}^{Q E}\left(\psi^{\prime}\right)+N f_{n}^{Q E}\left(\psi^{\prime}\right)\right] .
$$

The same consideration can be performed equivalently on the basis of the nucleon momentum distributions for protons $n^{p}(k)$ and neutrons $n^{n}(k)$ [5] and this is done using NMD from the LFD $[5,14,15]$.

In Figure 1 we give the QE CDFM scaling function for ${ }^{4} \mathrm{He},{ }^{12} \mathrm{C},{ }^{27} \mathrm{Al},{ }^{82} \mathrm{Kr}$ and ${ }^{197} \mathrm{Au}$ compared with experimental data and RFG results [4]. As can be seen our calculations explain very well the data for $\psi^{\prime}<0$ including $\psi^{\prime}<-1$ whereas in the RFG model $f\left(\psi^{\prime}\right)=0$ for $\psi^{\prime} \leq-1$.

In the present work we introduce phenomenologically the asymmetric shape of the scaling function, thus simulating the role of the final-state interactions (FSI), using a parameter $c_{1}$ which gives the correct maximum value of the scaling function and also an asymmetry in $f^{Q E}\left(\psi^{\prime}\right)$ for $\psi^{\prime} \geq 0$. One can see in Figure 2 the CDFM QE scaling function for different values of $c_{1}$ in comparison with empirical data and other calculations [9].

Our analysis within CDFM and LFD is extended to the $\Delta$-peak region weighting the RFG scaling function in the

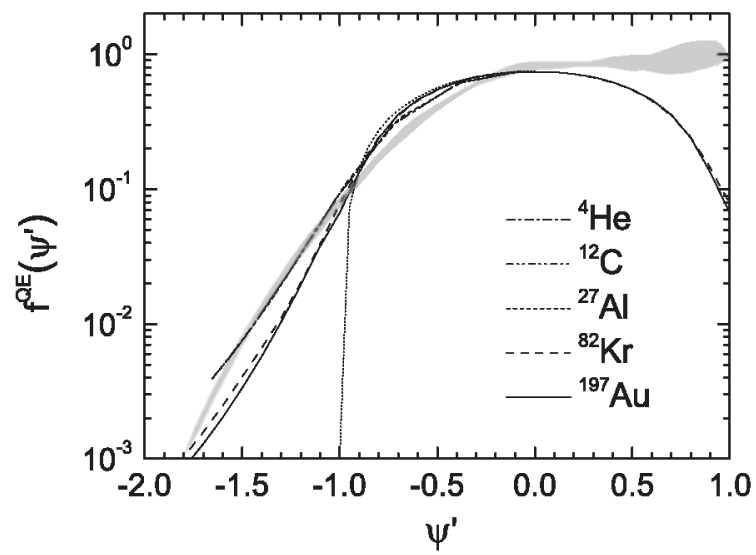

FIGURE 1. The quasielastic scaling function $f^{Q E}\left(\psi^{\prime}\right)$ at $q=1000 \mathrm{MeV} / \mathrm{c}$ for ${ }^{4} \mathrm{He},{ }^{12} \mathrm{C},{ }^{27} \mathrm{Al},{ }^{82} \mathrm{Kr}$ and ${ }^{197} \mathrm{Au}$ calculated in CDFM. Dotted line: RFG model result. Grey area: experimental data [4].

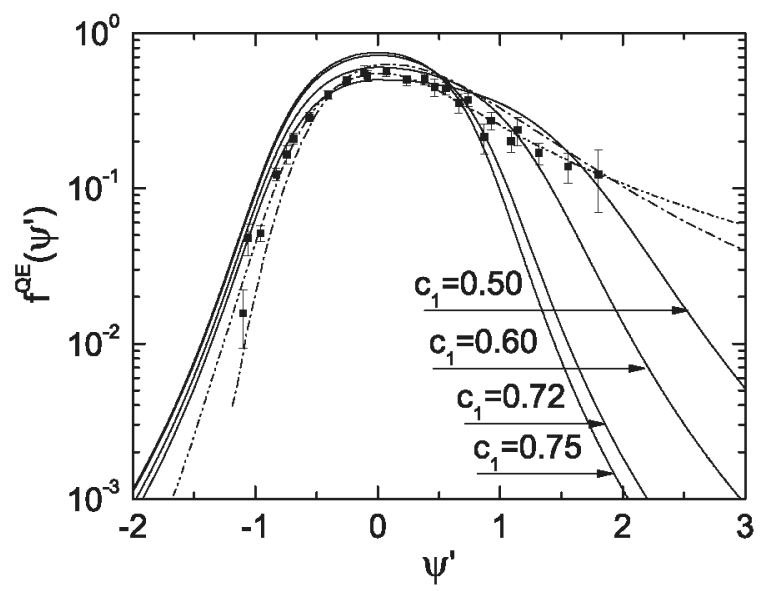

FIGURE 2. The quasielastic scaling function $f^{Q E}\left(\psi^{\prime}\right)$ for ${ }^{12} \mathrm{C}$ calculated in CDFM in comparison with the experimental data (black squares) [8]. CDFM results for different values of $c_{1}$ are presented by solid lines. Also shown for comparison is the phenomenological curve which fits the data (dash-two dots), as well as the curve that fits the $\left(e, e^{\prime}\right)$ results from [9] (dash-dot line)

$\Delta$-region by the CDFM weight function. The results of our work are presented in Figure 3.

\section{SCALING FUNCTIONS AND INCLUSIVE LEPTON SCATTERING}

In the one-photon-exchange approximation, the doubledifferential cross section in the laboratory system can be expressed by the longitudinal $R_{L}$ and transverse $R_{T}$ response functions which contain all the information on the distribution of the nuclear electromagnetic charge 


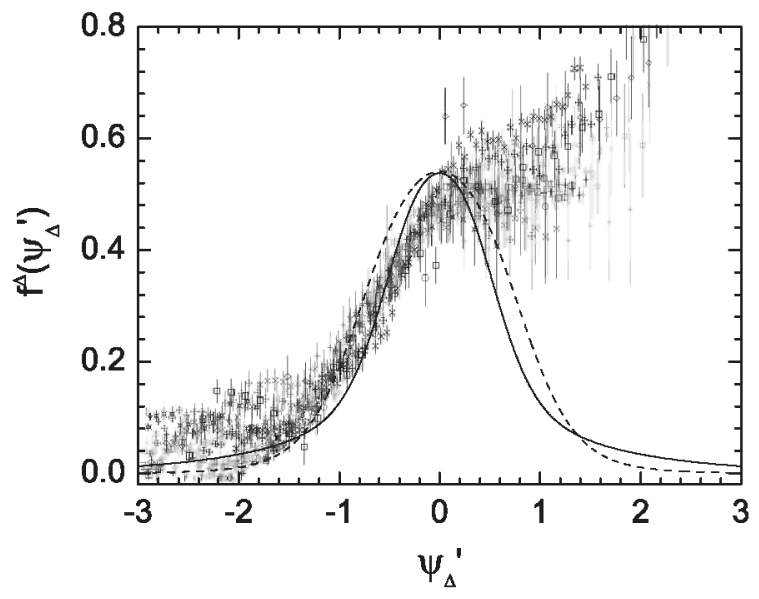

FIGURE 3. The $f^{\Delta}\left(\psi_{\Delta}^{\prime}\right)$ scaling function for ${ }^{12} \mathrm{C}$ in the $\Delta$ region. Dashed line: CDFM result (with $R_{\Delta}=1.565 \mathrm{fm}, b_{\Delta}=$ $0.420 \mathrm{fm}, k_{F}=1.20 \mathrm{fm}^{-1}$ ). Solid line: result of modified LFD approach $\left(\beta=0.80, k_{F}=1.20 \mathrm{fm}^{-1}\right)$. The coefficient $c_{1}=$ 0.54 in both CDFM and LFD cases. Averaged experimental values of $f^{\Delta}\left(\psi_{\Delta}^{\prime}\right)$ are taken from [8].

and current densities. These functions can be evaluated as components of the nuclear tensor $W_{\mu \nu}$. In [3] this tensor is computed in the framework of the RFG model. In this framework the nuclear response functions in both quasielastic $(X=Q E)$ and $\Delta$-resonance $(X=\Delta)$ regions have the general structure

$$
R_{X}=\frac{\mathscr{N} m_{N}}{q k_{F}}\left[R_{X}\right]^{\mathrm{s} n \mathrm{n} .} f_{\mathrm{RFG}}^{X}\left(\psi_{X}\right)
$$

where $\mathscr{N}=Z$ or $N,\left[R_{X}\right]^{\text {s.n. }}$ is the single-nucleon response function and $f_{\mathrm{RFG}}^{X}\left(\psi_{X}\right)$ is the QE- or $\Delta$ - scaling function. Within the CDFM in the expressions for the response functions $R_{L(T)}$ the RFG scaling functions are changed by the CDFM QE- and $\Delta$-scaling functions.

In Figure 4 we give an example of some results of calculations within the CDFM of inclusive electron scattering on ${ }^{12} \mathrm{C}$ at different incident energies and angles. The inclusive electron- ${ }^{12} \mathrm{C}$ scattering cross section is the sum of the $\mathrm{QE}$ and $\Delta$-contribution. The results of the CDFM calculations are presented for two values of the coefficient $c_{1}$ in the $\mathrm{QE}$ case (noted further by $c_{1}^{Q E}$ ), namely for $c_{1}^{Q E} \simeq 0.72$ and $c_{1}^{Q E}=0.63$. This is related to two types of experimental data. In the first one the transferred momentum in the position of the maximum of the QE peak extracted from data $\left(\omega_{\exp }^{Q E}\right)$ is $q_{\exp }^{Q E} \geq 450 \mathrm{MeV} / \mathrm{c} \simeq 2 k_{F}$ roughly corresponding to the domain where scaling is fulfilled (this is the case in Figure 4). In this case we found by fitting to the maximum of the QE peak the value of $c_{1}^{Q E}$ to be 0.73 . This leads to a weak asymmetry of the CDFM scaling function. In the second type of experimental data $q$ exp is not in the region where condition for

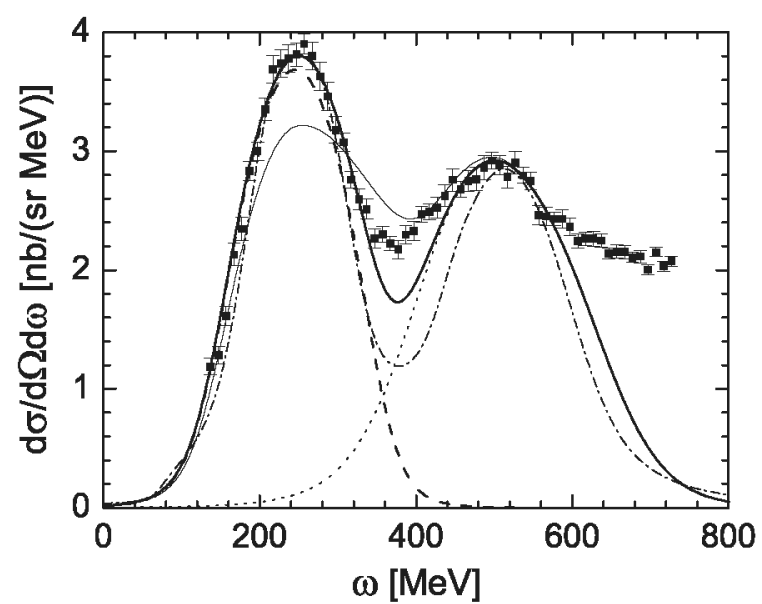

FIGURE 4. Inclusive electron scattering on ${ }^{12} \mathrm{C}$ at $\varepsilon=1108$ $\mathrm{MeV}$ and $\theta=37.5^{\circ}\left(q_{\exp }^{Q E} \simeq 675 \mathrm{MeV} / \mathrm{c}>2 k_{F}\right)$. The results obtained using $c_{1}^{Q E}=0.73$ in the CDFM scaling function for the $\mathrm{QE}$ cross section and the total result are given by dashed and thick solid line, respectively. Dotted line: using CDFM $\Delta$-scaling function; thin solid line: total CDFM result with $c_{1}^{Q E}=0.63$. Dot-dashed line: using $Q E$ - and $\Delta$-scaling functions obtained in the LFD approach. The experimental data are taken from [17].

scaling of the first kind is fulfilled $\left(q q_{\mathrm{exp}}^{Q E}<450 \mathrm{MeV} / \mathrm{c}\right)$. For them we found by fitting to the maximum of the QE peak the value of $c_{1}^{Q E}$ to be 0.63 . For these cases the CDFM scaling function is definitely asymmetric. One can see that the results for the almost symmetric CDFM scaling function agree with the electron data in the region close to the QE peak in cases where $q$ exp $\geq 450 \mathrm{MeV} / \mathrm{c}$. The results with asymmetric CDFM scaling function agree with the data in cases where $q \operatorname{eE}<450 \mathrm{MeV} / \mathrm{c}$. In Figure 4 we present also the calculations of the electron cross sections using QE- and $\Delta$ - scaling functions obtained by using the nucleon momentum distributions obtained in the LFD.

We present applications of the CDFM and LFD scaling functions to calculations of charge-changing neutrino-nucleus reaction cross sections. We follow the description of the formalism given in [8]. The chargechanging neutrino cross section in the target laboratory frame is given in the form

$$
\left[\frac{d^{2} \sigma}{d \Omega d k^{\prime}}\right]_{\chi} \equiv \sigma_{0} \mathscr{F}_{\chi}^{2},
$$

where $\chi=+$ for neutrino-induced reaction (e.g., $v_{\ell}+n \rightarrow \ell^{-}+p$, where $\left.\ell=e, \mu, \tau\right)$ and $\chi=-$ for antineutrino-induced reactions (e.g., $\bar{v}_{\ell}+p \rightarrow \ell^{+}+n$ ). The quantity $\mathscr{F}_{\chi}^{2}$ which depends on the nuclear structure contains [8] the nuclear response functions which are expressed in terms of the nuclear tensor $W^{\mu v}$ in both 
$\mathrm{QE}$ and $\Delta$-regions using its relationships with the RFG model scaling functions. In our work, instead of the RFG scaling functions in QE- and $\Delta$-regions, we use those obtained in the CDFM and LFD approach. In Figure 5 we present the results of calculations for cross sections of QE antineutrino $\left(\bar{v}_{\mu}, \mu^{+}\right)$scattering on ${ }^{12} \mathrm{C}$ for antineutrino energy $\varepsilon_{\bar{v}}=1 \mathrm{GeV}$. The results obtained by using the almost symmetric CDFM scaling function are close to the RFG model results, while those obtained with the use of asymmetric CDFM and LFD scaling functions are close to the predictions of the ERFG method $[8,16]$.

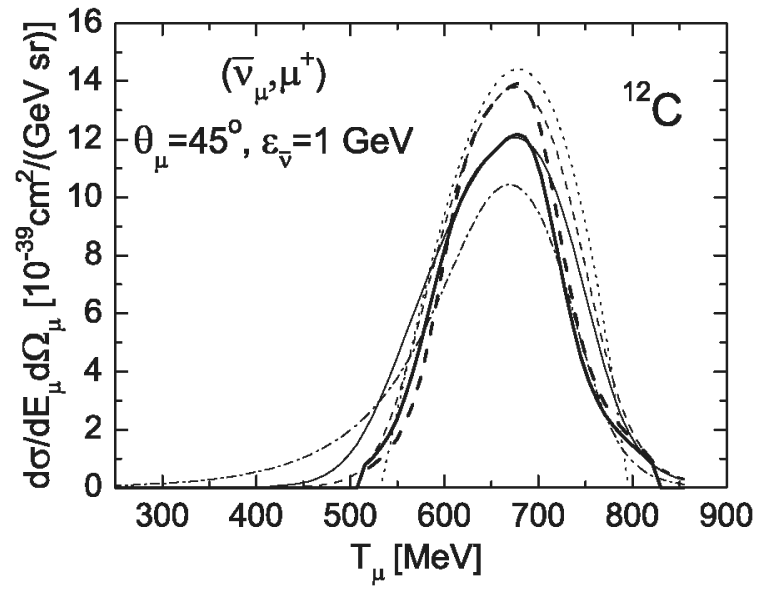

FIGURE 5. The cross section of quasielastic chargechanging $\left(\bar{v}_{\mu}, \mu^{+}\right)$reaction on ${ }^{12} \mathrm{C}$ for $\varepsilon=1 \mathrm{GeV}$ using QEscaling functions in CDFM (thin solid line: with $c_{1}=0.63$; thin dashed line: with $\left.c_{1}=0.72\right)$. The results using QE-scaling functions in LFD (thick solid line: with $c_{1}=0.63$; thick dashed line: with $c_{1}=0.72$ ) are also presented. The RFG model result and ERFG result $[8,16]$ are shown by dotted and dash-dotted lines, respectively.

\section{CONCLUSIONS}

The results of the present work can be summarized as follows: i) The quasielastic scaling function $f\left(\psi^{\prime}\right)$ is calculated in the CDFM equivalently by means of both density and nucleon momentum distributions for light, medium and heavy nuclei (including those with $Z \neq N$ for which proton and neutron densities are not similar): ${ }^{4} \mathrm{He},{ }^{12} \mathrm{C}$, ${ }^{27} \mathrm{Al},{ }^{56} \mathrm{Fe},{ }^{82} \mathrm{Kr},{ }^{118} \mathrm{Sn},{ }^{197} \mathrm{Au}$. The results explain the superscaling for $\psi^{\prime} \leq 0$ including $\psi^{\prime}<-1$ whereas in the RFG model $f\left(\psi^{\prime}\right)=0$ for $\psi^{\prime} \leq-1$; ii) Asymmetry in CDFM QE $f\left(\psi^{\prime}\right)$ is introduced phenomenologically, thus simulating the role of FSI which violate the symmetry for $\psi^{\prime} \geq 0$; iii) A realistic QE scaling function $f\left(\psi^{\prime}\right)$ is obtained also on the basis of NMD's $n(k)$ calculated within the modified light-front dynamics method; iv) The CDFM and LFD approaches are extended successfully to the $\Delta$-peak region which is the main contribution to the inelastic scattering; v) The QE- and $\Delta$-scaling functions in CDFM and LFD are applied to description of data on inclusive electron scattering by ${ }^{12} \mathrm{C}$ at large energies and transferred momenta. The question of almost symmetric or asymmetric $f\left(\psi^{\prime}\right)$ is considered in relation to the value of $q_{\exp }^{Q E}$ in the position of the maximum of the $\mathrm{QE}$ peak extracted from data $\left(\omega_{\text {exp }}^{Q E}\right)\left(\geq 450 \mathrm{MeV} / \mathrm{c} \simeq 2 k_{F}\right.$ or $<450 \mathrm{MeV} / \mathrm{c}$ ); vi) The CDFM and LFD scaling functions (the same from the $\left(e, e^{\prime}\right)$ analysis) are applied to calculations of charge-changing neutrino- ${ }^{12} \mathrm{C}\left(v_{\mu}, \mu^{-}\right)$ and $\left(\bar{v}_{\mu}, \mu^{+}\right)$reaction cross sections for energies of the incident particles from 1 to $2 \mathrm{GeV}$. The results are compared with those of RFG and ERFG methods.

\section{ACKNOWLEDGMENTS}

This work was partly supported by the Bulgarian National Science Foundation under Contracts No.Ф1416 and $\Phi-1501$ and by funds provided by DGI of MCyT (Spain) under Contract Nos. FIS 2005-00640, BFM 2003-04147-C02-01, INTAS-03-54-6545, FPA 2005-04460, and FIS 2005-01105.

\section{REFERENCES}

1. G. B. West, Phys. Rep. 18, 263-323 (1975).

2. D. B. Day et al., Annu. Rev. Nucl. Part. Sci. 40, 357-409 (1990); C. Ciofi degli Atti et al., Phys. Rev. C 36, 1208 1211 (1987); ibid. 43, 1155-1176 (1991); C. Ciofi degli Atti, and G. B. West, Phys. Lett. B458, 447-453 (1999).

3. W. M. Alberico et al., Phys. Rev. C 38, 1801-1810 (1988).

4. T. W. Donnelly, and I. Sick, Phys.Rev. Lett. 82, 3212-3215 (1999); Phys. Rev. C 60, 065502 (1999).

5. A. N. Antonov et al., Phys. Rev. C 69, 044321 (2004); ibid. 71, 014317 (2005); ibid. 73, 047302 (2006).

6. M. B. Barbaro et al., Nucl. Phys. A643, 137-160 (1998).

7. C. Maieron et al., Phys. Rev. C 65, 025502 (2002).

8. J. E. Amaro et al., Phys. Rev. C 71, 015501 (2005).

9. J. A. Caballero et al., Phys. Rev. Lett. 95, 252502 (2005); J.A. Caballero, Phys. Rev. C 74, 015502 (2006).

10. J. E. Amaro et al., Phys. Rev. C 73, 035503 (2006)

11. C. Maieron et al., Phys. Rev. C 68, 048501 (2003).

12. A. Meucci, C. Giusti, and F. D. Pacati, Nucl. Phys. A739, 277-290 (2004); ibid. A744, 307-322 (2004); ibid. A773, 250-262 (2006).

13. A. N. Antonov, P. E. Hodgson, and I. Zh. Petkov, Nucleon Correlations in Nuclei, Springer-Verlag, BerlinHeidelberg-New York, 293 pp., 1993 (and references therein)

14. A. N. Antonov et al., Phys. Rev. C 65, 024306 (2002).

15. J. Carbonell, and V. A. Karmanov, Nucl.Phys. A581, 625-653 (1995); J. Carbonell et al., Phys. Rep. 300, 215-347 (1998).

16. M. B. Barbaro et al., Phys. Rev. C 69, 035502 (2004).

17. R. M. Sealock et al., Phys. Rev. Lett. 62, 1350-1353 (1989).

18. D. T. Baran et al., Phys. Rev. Lett. 61, 400-403 (1988). 